OPEN ACCESS

Edited by:

Kevin R. Brooks,

Macquarie University,

Australia

Reviewed by:

Min Bao,

Chinese Academy of Sciences, China

Marco Turi,

Fondazione Stella Maris Mediterraneo

Onlus, Italy

Katherine Rebecca Storrs,

Justus Liebig University Giessen,

Germany

${ }^{*}$ Correspondence: Matthew R. Longo

m.longo@bbk.ac.uk

Specialty section:

This article was submitted to

Perception Science,

a section of the journal

Frontiers in Psychology

Received: 13 May 2019 Accepted: 01 November 2019

Published: 21 November 2019

Citation:

Ambroziak KB, Azañón E and

Longo MR (2019) Body Size

Adaptation Alters Perception of Test Stimuli, Not Internal Body Image.

Front. Psychol. 10:2598.

doi: 10.3389/fpsyg.2019.02598

\section{Body Size Adaptation Alters Perception of Test Stimuli, Not Internal Body Image}

\author{
Klaudia B. Ambroziak ${ }^{1,2}$, Elena Azañón ${ }^{3,4,5}$ and Matthew R. Longo ${ }^{1 *}$ \\ ${ }^{1}$ Department of Psychological Sciences, Birkbeck, University of London, London, United Kingdom, ${ }^{2}$ School of Advanced \\ Study, The Warburg Institute, University of London, London, United Kingdom, ${ }^{3}$ Department of Experimental Psychology, \\ Institute of Psychology II, Otto-von-Guericke University, Magdeburg, Germany, ${ }^{4}$ Center for Behavioral Brain Sciences, \\ Magdeburg, Germany, ${ }^{5}$ Department of Behavioral Neurology, Leibniz Institute for Neurobiology, Magdeburg, Germany
}

Recent studies have reported that adaptation to extreme body types produces aftereffects on judgments of body normality and attractiveness, and also judgments of the size and shape of the viewer's own body. This latter effect suggests that adaptation could constitute an experimental model of media influences on body image. Alternatively, adaptation could affect perception of test stimuli, which should produce the same aftereffects for judgments about participant's own body or someone else's body. Here, we investigated whether adaptation similarly affects judgments about one's body and other bodies. We were interested in participants' own body image judgments, i.e., we wanted to measure the mental representations to which the test stimuli were compared and not the perception of test stimuli per se. Participants were adapted to pictures of thin or fat bodies and then rated whether bodies were fatter or thinner than either: their own body, an average body (Experiment 1), or the body of another person (Experiments 2 and 3). By keeping the visual stimuli constant but changing the task/type of judgment, i.e., the internal criterion participants are asked to judge the bodies against, we investigated how adaptation affects different stored representations of bodies, specifically own body image vs. representations of others. After adaptation, a classic aftereffect was found, with judgments biased away from the adapting stimulus. Critically, aftereffects were nearly identical for judgments of one's own body and for other people's bodies. These results suggest that adaptation affects body representations in a generic way and may not be specific to the own body image.

Keywords: body representations, visual perception, sensory adaptation, self, aftereffects

\section{INTRODUCTION}

In our daily lives, we constantly experience our own bodies directly through touch and proprioception, but most of our visual experience with bodies comes from seeing other people. For most of us, other people's bodies are a ubiquitous part of our "visual diet" (Boothroyd et al., 2012). Moreover, through the media, we are bombarded with images of other, often idealized, bodies. Previous research showed that exposure to thin, idealized images changes attitudes toward one's own body, increases body dissatisfaction, and negatively affects mood 
(Groesz et al., 2002; Tiggemann and McGill, 2004). Clearly, media can shape our beliefs and attitudes, but can exposure to certain body types change the way we actually perceive our own bodies? Recently, several studies suggested that this might be the case and proposed visual adaptation, i.e., a shift in perceptual judgment after prolonged exposure to a certain stimulus, as one of the mechanisms that may be involved in this process (Hummel et al., 2012b; Brooks et al., 2016; Bould et al., 2018; Stephen et al., 2018). According to this emerging theory, exposure to idealized, often extremely thin bodies (e.g., "size zero" models) causes our own body to be perceived as fatter than it really is. Alternatively, adaptation to extreme body types might affect the way people visually perceive test stimuli, without affecting the viewer's own body image at all. Here, we further investigated whether short-term effects of adaptation could constitute an experimental model for the long-term effects of media influences on body image, by asking whether perceptual changes induced by body size adaptation show specificity to one's own body or, on the contrary, are similar across visual representations of bodies in general. We were interested in body image judgments, i.e., we wanted to measure the mental representations to which the test stimuli were compared.

Body representations arise from many different sources: interoceptive signals from internal organs; sensory input from the external world coming from modalities such as touch and vision; and also from abstract knowledge, beliefs, and attitudes (see Longo et al., 2010 for a review). The body image is a complex concept that involves both sensory and cognitive components. While many body representations are thought to be largely based on somatosensory signals about touch, proprioception, pain, etc. (Longo, 2015), the body image is thought to be predominantly visual. Indeed, in his classic definition, Schilder (1935/1950) used overtly visual language in describing the body image as "the picture of our own body which we form in our mind, that is to say the way in which the body appears to ourselves" (p. 11). Although, in the literature, body image is often used in reference to attitudes and feeling about one's body, here we wanted to specifically focus on its visual component, i.e., the way the body appears to us in the mind's eye. As a predominantly visual representation, is the body image then shaped by visual exposure to other bodies?

Visual adaptation produces aftereffects which bias perception in the direction opposite to the adapting stimulus. For example, in the classic waterfall illusion, after a short exposure to the flowing water of a waterfall, when the observer looks at a static image, e.g., the space beside the waterfall, she will perceive it as moving upward (Barlow and Hill, 1963; Anstis et al., 1998). Visual adaptation aftereffects are widely studied and well established for basic features, such as motion (Wohlgemuth, 1911), orientation (Gibson and Radner, 1937), and color (McCollough, 1965), which are thought to be processed mainly at lower levels of visual hierarchy. However, research on adaptation to complex stimuli such as faces and bodies suggests that adaptation is not limited to basic features and may operate at higher levels of processing (Rhodes et al., 2003; Webster et al., 2004; Fox and Barton, 2007; Pond et al., 2013; Brooks et al., 2018). Research on face adaptation shows that adaptation to faces with consistent distortions, i.e., compressing or expanding the center of a face, causes normal faces to appear distorted in the opposite direction (Rhodes et al., 2003). In a similar way, gender-ambiguous faces are judged as more masculine after prolonged viewing of female faces, whereas adaptation to male faces induces the opposite effect (Webster et al., 2004; Pond et al., 2013). Facial emotional expressions also produce similar aftereffects: after exposure to happy faces, observers tend to perceive subsequent neutral expressions as sad (Fox and Barton, 2007).

Research on body adaptation has shown that brief exposure to unfamiliar thin bodies significantly alters people's perception of body attractiveness, normality, and ideals, in the direction of the thin adaptor (Winkler and Rhodes, 2005; Glauert et al., 2009). Moreover, adaptation to participants' own bodies, depicting them as either thinner or fatter, can also alter the way participants judge images of their own bodies. After adaptation to the thin version of their own body, participants rated a thinner than actual image to be the most accurate depiction of their own body and vice versa for the fat adaptor (Hummel et al., 2012a,b, 2013). Interestingly, the effect of body adaptation transfers across identities, with comparable effects for unfamiliar and own body adaptors (Hummel et al., 2012b; Brooks et al., 2016). Moreover, it is specific to bodies, and does not transfer between bodies and narrow/wide rectangles Hummel et al. (2012a). Taken together, these findings show that exposure to thin images not only affects perceived norms and ideals (Winkler and Rhodes, 2005; Glauert et al., 2009) but can also change how participants judge images of their own body - causing the actual image of the participant's body to appear fatter (Hummel et al., 2012b; Brooks et al., 2016). These results suggest that visual adaptation may serve as an experimental model of the effect that exposure to thin bodies presented in media has on body image. Following this line of reasoning, Challinor et al. (2017) proposed that including visual adaptation as a part of treatment may have therapeutic effect on patients with body image distortions in conditions such as anorexia nervosa.

Here, we investigated whether adaptation affects visual body image in a self-specific way. There is clear evidence that body size adaptation changes perception of bodies as indicated by the shift in perceived norms as the result of aftereffects. The question nevertheless remains how similar the magnitude of the aftereffects is for one's own body (i.e., the body image) and for bodies in general. Specifically, it is not known whether exposure to bodies affects judgments about our own body in a self-specific way, or the effect is generic to all bodies. For visual adaptation to constitute an experimental model of media effect on body image distortions, some overlap in the way adaptation affects one's own body and bodies of others is required to allow the transfer of aftereffects from media images to body image as it has been argued previously (Brooks et al., 2016). However, the effect that adaptation has on one's own body should also differ from the general effect of adaptation 
on all images of bodies. If adaptation affects all bodies equally, the relative difference between one's own body and other bodies should not change. In other words, both our own bodies and bodies presented in media should be affected by adaptation. In consequence, our own body should not appear to us as fatter in the whole continuum of bodies, i.e., if we were to compare our body to other bodies. Interestingly, there are many clinical conditions in which people have distorted image of their own body, but not other people's (whereas the opposite is rare). Studies have reached divergent conclusions about whether patients with eating disorders selectively overestimate the size of their own bodies (e.g., Mizes, 1992; Øverås et al., 2014) or overestimate bodies in general (e.g., Tovée et al., 2000; Horndasch et al., 2015). In a study by Guardia et al. (2012), patients showed biased judgments about their own actions but could accurately judge the affordances of others. These results suggest that anorexia nervosa patients do not have distorted representations of other people's bodies.

To investigate whether visual exposure to extreme body types affects the perception of our own body and of other bodies in similar or different ways, we designed three experiments in which female participants judged the same test images but compared them to different internal representations. After adaptation to pictures of either extremely thin or extremely fat bodies, participants were asked to rate whether subsequently presented bodies were fatter or thinner than either: their own body (all three experiments), an average body (Experiment 1), or a body of a specific other person (Experiments 2 and 3). By keeping the visual stimuli constant but changing the internal criterion (or anchor point) that participants are asked to judge the bodies against, we investigated how adaptation affects different stored representations of bodies, specifically, and not the perception of the test stimuli per se. Note that in a body image judgment, the reference (a photograph, a silhouette, or even a piece of string) is usually compared to one's mental representation of one's own body, regardless of the nature of the reference itself (e.g., Slade and Russell, 1973; Garner et al., 1976; Thompson et al., 1986; Schneider et al., 2009). Interestingly, a previous study by de la Rosa et al. (2014) reported modulation of action adaptation aftereffects across two conditions that had identical adapting and test stimuli but differed with respect to the information that was provided prior to the adaptation, i.e., the social context, suggesting that the context of the task can affect adaptation aftereffects.

\section{EXPERIMENT 1}

In Experiment 1, we investigated the effect of visual adaptation to thin bodies on judgments about one's own body and about an average body. If adaptation has a specific effect on body image, the magnitude of adaptation aftereffects should differ depending on whether participants are comparing the test image to their own body or somebody else's. If, in contrast, adaptation affects body image judgments by altering perception of the test stimuli, identical aftereffects should be found in both cases.

\section{Methods}

\section{Participants}

Due to the nature of our stimuli (depicting female bodies), we restricted our sample to female participants. A different group of participants was selected in each study. Twenty participants (mean age: 28.1, SD: 11.6, range: 18-65; mean body mass index, BMI: 21.9, SD: 3.1, range 17.9-29.1) took part in Experiment 1. All participants had normal or corrected to normal vision. All participants gave informed consent and were paid for their participation. The procedures were approved by the ethics board of the Department of Psychological Sciences, Birkbeck, University of London.

Previous studies showed that adaptation to bodies produces strong and robust effects. For example, Hummel et al. (2012b) using similar type of stimuli and similar type of judgments in two experiments obtained Cohen's $d_{\mathrm{z}}$ 's of 2.87 and 1.04 . We conducted a power analysis using $\mathrm{G}^{\star}$ Power 3.1 taking the smaller of these two effect sizes, an alpha value of 0.05 and power of 0.95 , which indicated that 12 participants were required. In addition, piloting data using our paradigm showed adaptation effects in virtually every participant. Thus, we believe that our sample size of 20 participants makes this experiment well powered to address this issue.

\section{Stimuli}

We used a set of 89 images of female bodies rendered from $3 \mathrm{D}$ avatars generated in DAZ Studio 4.8 (DAZ Productions, http://www.daz3d.com/). Avatars' BMI ranged from 13 (emaciated) to 35 (obese) with an increment of $0.25 \mathrm{BMI}$ units between each stimulus (with a total of 89 images; see Figure 1 for examples). To approximate the avatars' BMI, we used Cornelissen et al.s (2009) formula to calculate the waist-to-hip ratio (WHR) for white $\mathrm{UK}$ women of reproductive age: $\mathrm{WHR}=(2.057 \times \mathrm{BMI}+29.67) /(1.842 \times \mathrm{BMI}+56.004)$, which is based on data from Health Survey for England (2003). Following this formula, waist and hip circumferences were estimated for each required BMI from the range of 13-35. Avatars' waist and hip circumferences were adjusted using the Universal Sizing Apparatus tool (Rocketship Technologies Inc., http://rocketship3d.com/). The height of the avatars was kept constant at $170 \mathrm{~cm}$. The avatars were rotated approximately $45^{\circ}$ around the vertical axis (in the transverse plane) to obtain a viewing angle that would provide more information about the body dimensions compared to the straight-facing view. A recent study by Cornelissen et al. (2018) confirmed that this three-quarter view results in the most accurate estimations of body size. Finally, 2D images were rendered from the avatars, as shown in Figure 1.

\section{Procedures}

Each experiment consisted of the Baseline and the Adaptation phase conducted on the same day with a short break (1-2 min). In Experiments 1 and 2, a very thin body (BMI = 13) was used as an adapting stimulus. In Experiment 3, the procedure was repeated twice, using both a very thin $(\mathrm{BMI}=13)$ and a very fat adaptor $(\mathrm{BMI}=35)$. In all experiments, participants 


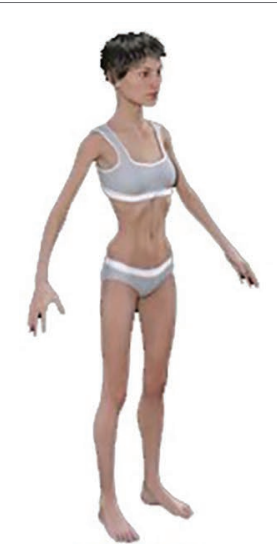

BMI 13
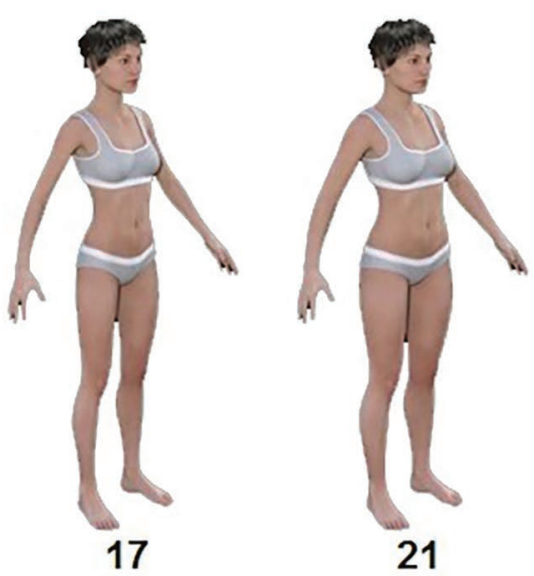
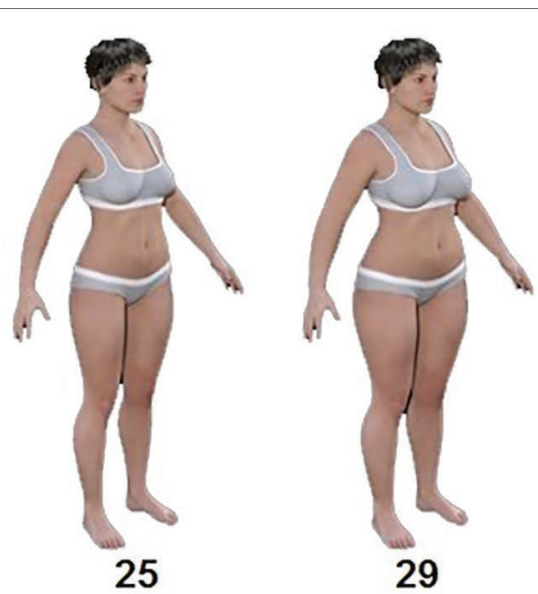

FIGURE 1 | Stimuli used in the experiments. A continuum of 89 body shapes was created, ranging from extremely thin (i.e., BMI = 13) to obese (i.e., BMI = 35). 3D modeling software was used to model these changes in a biologically realistic way. The numbers indicate the estimated body mass index (BMI) of the avatars.

sat approximately $50 \mathrm{~cm}$ from the screen with head movements unrestricted. Images were presented in the center of a 24 -inch screen (resolution: 1,600 $\times 1,200$ pixels; refresh rate: $75 \mathrm{~Hz}$ ), on a black background. The height of each image was $18 \mathrm{~cm}$ $\left(20^{\circ}\right.$ visual angle). Stimuli were presented using Psychtoolbox (Brainard, 1997), running on MATLAB (Mathworks, Natick, MA, USA). The PSEs were calculated using a Bayesian adaptive algorithm QUEST (Watson and Pelli, 1983). Statistical analyses were performed in JASP (JASP Team, 2017).

In the Baseline phase, each trial began with a blank screen $(1,000 \mathrm{~ms})$, followed by a fixation cross $(1,000 \mathrm{~ms})$ that turned from black to red to indicate the beginning of a new trial. Then, a question appeared on the screen: Is this body fatter than your own? in the Self condition, or Is this body fatter than average? in the Average condition. Before the start of the task, we explained to participants that "average" means the most common/typical body for their age and gender (according to their best guess). The question was followed by a 1,000-ms test body selected by QUEST, from a set of possible stimuli based on their history of responses on previous trials. A blank screen remained visible until the response was made using labeled keyboard keys ("yes"/ "no"). After the response, a black cross appeared on the screen for 1,000 ms indicating the end of the trial. Each part was divided in four blocks of 40 trials (two blocks per condition, ABBA order counterbalanced across participants). At the end of each block, the point of subjective equality (PSE, i.e., the stimulus for which the participant was equally likely to judge it as fatter or thinner) was calculated using QUEST. The PSEs from the two blocks were then averaged to obtain a single estimate for each condition, separately for the Baseline and the Adaptation phase.

The Adaptation phase started with an initial 2-min exposure to the thin adaptor. The adaptor flickered every $4 \mathrm{~s}$ (disappearing for $500 \mathrm{~ms}$ and appearing again) to maintain attention. After that, participants performed the same task as in the Baseline phase. Each trial in the Adaptation phase was identical to the
Baseline with the addition of a thin body exposure. A very thin adaptor (BMI 13) was presented for $8 \mathrm{~s}$ to "top-up" the adaptation, followed by one second of blank screen, just before the presentation of the corresponding question (i.e., is this body fatter than your own/average) and the test stimulus. Again, the PSE was calculated after every block, resulting in two PSEs per condition.

\section{Results}

In each experimental session, two 50\% thresholds (PSEs) per condition were calculated using QUEST to estimate the BMI at which participants were equally likely to respond thinner or fatter. These two PSEs were then averaged, resulting in four PSEs, one for each condition (Self, Average) and adaptation phase (Baseline and Adaptation). Our main effect of interest was the pre-post adaptation shift of the PSEs in Self and Other condition. The results are shown in Figure 2 (left panel). Clear adaptation aftereffects were apparent in both conditions. In the Self condition, the mean perceived BMI decreased from 28.1 $(\mathrm{SD}=2.3)$ at pre-test to 25.9 $(\mathrm{SD}=2.8)$ after adaptation, Similarly, in the Average condition, mean judgments decreased from $28.5(\mathrm{SD}=1.9)$ at pre-test to $26.3(\mathrm{SD}=2.2)$ after adaptation. This clear decrease in perceived BMI from Baseline to Adaptation was significant, both in the Self condition (mean change: 2.22, SD: 1.24), $t(19)=8.05, p<0.001, d_{z}=1.80$, and in the Average condition (mean change: 2.26, SD: 1.68), $t(19)=6.00$, $p<0.001, d_{\mathrm{z}}=1.34$.

To investigate the effects of the two judgment types, we conducted a $2 \times 2$ repeated measures analysis of variance (ANOVA) on the PSEs with factors condition (Self / Average) and adaptation (Baseline / Adaptation). We found a main effect of adaptation, $F(1,19)=50.78, p<0.001, \eta_{\mathrm{p}}^{2}=0.75$. That is, after adaptation to a thin adaptor, participants perceived as fatter, images that before adaptation were considered as thinner (see Figure 2). There was no effect of condition, $F(1,19)=0.74, p=0.4, \eta_{\mathrm{p}}^{2}=0.04$, suggesting that on 

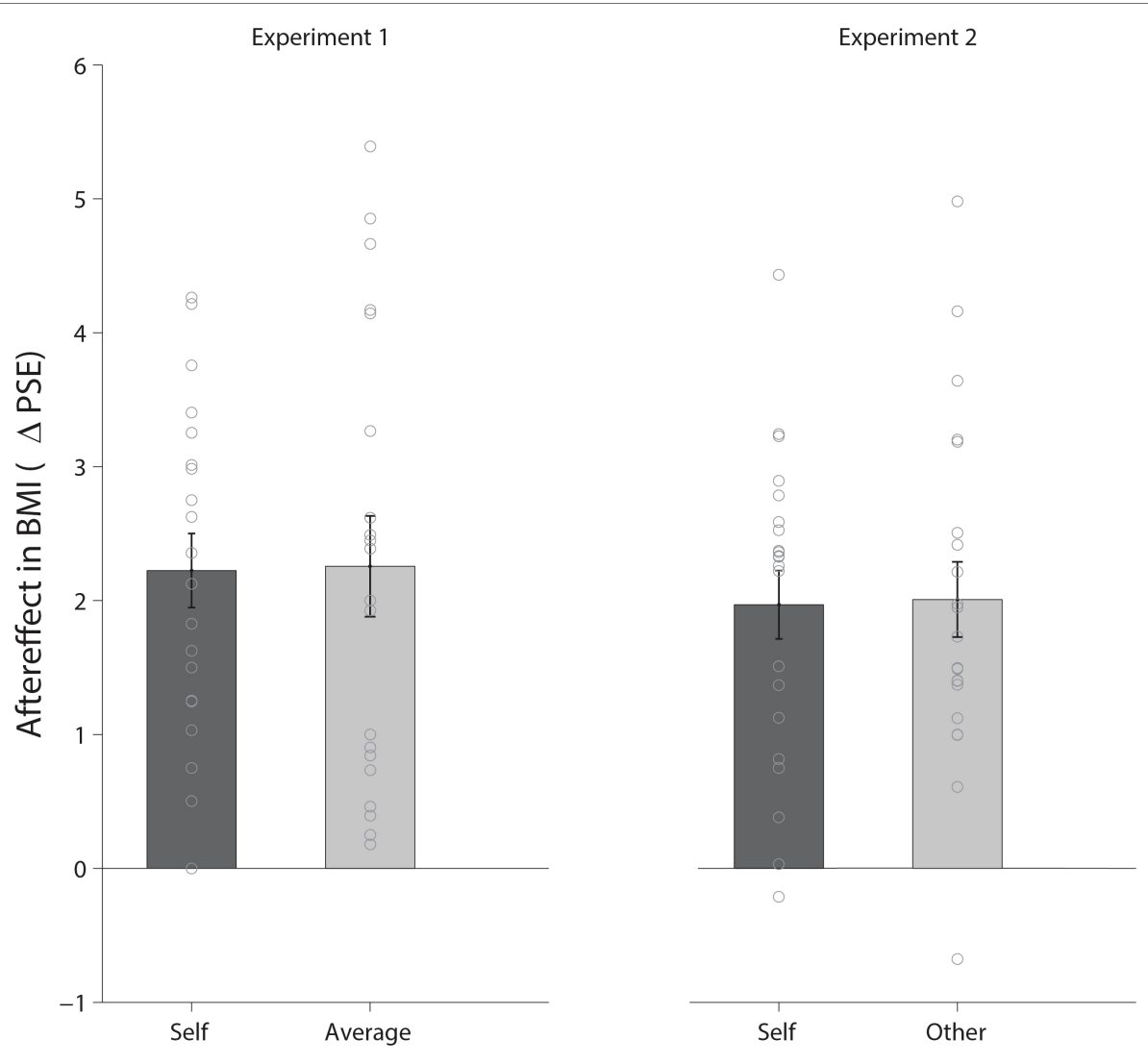

FIGURE 2 | Results of Experiments 1 (left panel) and 2 (right panel). (Left panel) The effect of adaptation for the Self and Average conditions shown as the pre-post adaptation shift in PSE. The dots indicate individual subjects, and the error bars represent standard errors. Clear adaptation aftereffects were apparent for both body image judgments in the Self condition and judgments of typicality in the Average condition. The magnitude of the aftereffects was very similar in the two conditions. (Right panel) The effect of adaptation for the Self and Other conditions presented in the same way as in Experiment 1.

average participants did not judge themselves as fatter or thinner than a typical woman. Critically, there was no interaction, $F(1,19)=0.026, p=0.874, \eta_{\mathrm{p}}^{2}=0.00$, suggesting that adaptation affected participants' perception of themselves in the same way it affected perception of typicality. Moreover, the magnitude of the aftereffects in both conditions was correlated across participants, $r=0.85, p<0.001$.

There was a correlation between participants' own BMI and the baseline PSEs in the Self condition: $r=0.71, p<0.001$, but not in the average condition: $r=0.16, p=0.500$. Similarly, after adaptation, there was a correlation between participants' own BMI and PSEs in the Self condition: $r=0.69, p<0.001$, but not in the Other condition: $r=0.35, p=0.128$. The clear correlation between actual and perceived BMI is important to note given that other tasks purporting to measure body image (such as the moving caliper method) have been criticized on the basis that no such correlations were apparent (e.g., Ben-Tovim and Crisp, 1984; Ben-Tovim et al., 1990).

To further compare the magnitude of the aftereffects in two conditions, we conducted a Bayesian paired $t$-test (Rouder et al., 2009), comparing the PSE change (Baseline/Adaptation) between conditions, which provided support for the null hypothesis, $\mathrm{BF}_{(0,0.7)}=0.24$.
The absence of an overall effect of Self vs. Average condition may suggest that participants genuinely considered themselves as being about the average size, despite the fact that the average BMI of our participants (21.9) was lower than the UK average for females in the same age range, which was reported to be 25.9 (Health Survey for England, 2016). Alternatively, the results may suggest that participants did not perform different tasks in the two conditions and possibly used themselves as a reference in both. Experiment 2 aimed to address these issues.

\section{EXPERIMENT 2}

The results of Experiment 1 revealed no differences between the Self and Average conditions. This therefore did not allow us to conclude that participants actually performed two different tasks in those two conditions. In Experiment 2, instead of the Average condition we designed a condition (Other), in which participants had to compare test stimuli with a specific person, namely the experimenter (KBA) with whom participants interacted prior to performing the task. We reasoned that the use of a specific person rather than an abstract "average" person would make the task easier and clearer to the participants. 


\section{Methods}

\section{Participants}

Twenty-one participants (mean age: 29.9 , SD: 10, range: 19-58; mean BMI: 23.7, SD: 4.8, range 17.0-35.0) took part in Experiment 2. All participants had normal or corrected to normal vision. All participants gave informed consent and were paid for their participation. The procedures were approved by the ethics board of the Department of Psychological Sciences, Birkbeck, University of London.

\section{Stimuli and Procedures}

Stimuli were identical to Experiment 1. Procedures were similar to Experiment 1. This time, however, participants were asked to compare the images either with themselves, answering the question: Is this body thinner or fatter than your own?, or with the experimenter (KBA), answering the question: Is this body thinner or fatter than Klaudia? (Other condition). Before the start of the experiment, participants had approximately $5 \mathrm{~min}$ of visual experience of the experimenter while she introduced the task. The experimenter was wearing close-fitting clothes and after explaining the task, she stood in front of the participant and asked them to memorize her body size and shape for about 10 s. During the task, participants were not looking at the experimenter. As in Experiment 1, the experiment consisted of two parts: Baseline and Adaptation. Each part was divided into four blocks of 30 trials (two blocks per Self/Other condition, in $\mathrm{ABAB}$ order, counterbalanced across participants). To shorten the length of the experiment, the duration of the adapting stimuli was reduced to $6 \mathrm{~s}$, and the duration of the initial blank screen and the fixation cross before and after the response was reduced to $500 \mathrm{~ms}$ each.

\section{Results and Discussion}

Results from Experiment 2 are shown in Figure 2 (right panel). In the Self condition, mean perceived BMI decreased from $25.7(\mathrm{SD}=3.4)$ at pre-test to $23.7(\mathrm{SD}=3.8)$ after adaptation. Similarly, in the Other condition, PSEs decreased from 24.3 $(\mathrm{SD}=1.7)$ at pre-test to $22.3(\mathrm{SD}=1.9)$ after adaptation. Again, clear adaptation aftereffects were significant in both the Self condition (mean change: 1.97, SD: 1.17$), t(20)=7.71$, $p<0.001, d_{z}=1.68$, and the Other condition (mean change: 2.01, SD: 1.29), $t(20)=7.15, p<0.001, d_{z}=1.56^{1}$. A $2 \times 2$ repeated measures ANOVA with factors condition and adaptation revealed a main effect of adaptation, $F(1,20)=81.47, p<0.0001$, $\eta_{\mathrm{p}}^{2}=0.80$, showing that exposure to a thin body affected the perception of test bodies. We also found a significant main effect of condition, $F(1,20)=5.30, p=0.032, \eta_{\mathrm{p}}^{2}=0.21$, which reflects the fact that the experimenter was perceived differently (overall as thinner) than the participants perceived

${ }^{1}$ Because of a programming error, in some subjects, some of the PSEs that came close to the upper limit of possible stimuli could have suffered from a ceiling effect. In all cases, only one PSE per condition was affected (two were obtained), which made it possible to remove the affected PSEs in an additional analysis. Removing the affected PSEs (10.6\% of all PSEs) did not change the overall pattern of the results. Imputing the affected PSEs using regression analysis also yielded very similar results. themselves. This difference between the two types of judgments is important as it demonstrates that participants were in fact making different judgments in the two conditions. Critically, however, there was no significant interaction, $F(1,20)=0.02$, $p=0.9, \eta_{\mathrm{p}}^{2}=0.00$, again suggesting that adaptation affected participants' perception of themselves in the same way it affected perception of the experimenter's body. As in Experiment 1, there was a positive correlation between the magnitude of the aftereffects in the two conditions, though it did not differ significantly from $0, r=0.35, p=0.12$.

There was a correlation between participants' own BMI and the baseline PSEs in the Self condition: $r=0.69, p<0.001$, but not in the Other condition: $r=0.08, p=0.730$. After adaptation, there was a correlation between participants' own BMI in both conditions: $r=0.72, p<0.001$ in the Self condition and $r=0.49, p=0.024$ in the Other condition, respectively.

As in Experiment 1, a Bayesian paired $t$-test comparing the magnitude of the change in PSE in the two conditions provided additional support for the null hypothesis: $\mathrm{BF}_{(0,0.7)}=0.23$, further suggesting that there is no difference in the magnitude of aftereffects between the Self and the Other condition.

These results showed that adaptation to an extremely thin body affected judgments about self vs. other body similarly and therefore the magnitude of the aftereffects was not influenced by the type of judgment being made. However, previous research suggested that in some cases body adaptation may be affected by the task. Winkler and Rhodes (2005) showed that while adaptation to a thin body had an effect on both perceived normality and attractiveness of test bodies, adaptation to a fat body did not significantly affect perceived attractiveness. In Experiment 3, we tested whether adaptation to fat bodies affected self vs. other body judgments equally.

One possible limitation of Experiment 2 is the fact that the experimenter was used as a reference in the Other condition. Although the pattern of results in this condition resembles typical adaptation aftereffects, it is possible that the responses were affected by some form of participant bias or social desirability bias in which participants change their responses to more socially acceptable. Since judging another person's weight is a sensitive task and participants knew that the experimenter would eventually see their responses, it is possible that they altered their judgments. Therefore, in Experiment 3, in which we tested the effect of both thin and fat exposure, we used a famous person (Kate Middleton) as a reference in the Other condition.

\section{EXPERIMENT 3}

Experiment 3 aimed to test the effect of thin and fat adaptation on judgments about self and other bodies. This time, in the Other condition, we used a famous person in the United Kingdom, i.e., Kate Middleton (the Duchess of Cambridge). We chose Kate Middleton as we expected that she would be familiar to a largest group of potential participants. In addition, $\mathrm{KM}$ has a BMI of about 18 which is lower than 
that of an average UK female (and indeed lower than that of 90\% of participants in Experiments 1 and 2), which made the difference between the conditions most apparent.

\section{Methods}

\section{Participants}

Eighteen participants took part in Experiment 3 (mean age: 27.1, SD: 7.3, range: 20-41; mean BMI: 20.2, SD: 2.2, range 17.0-25.4). Two additional participants who signed up for Experiment 3 were tested but their data were never analyzed. One of them was pregnant and the other one had a BMI beyond the range of our stimuli. All participants had normal or corrected to normal vision. All participants gave informed consent and were paid for their participation. The procedures were approved by the ethics board of the Department of Psychological Sciences, Birkbeck, University of London.

\section{Stimuli and Procedures}

Procedures were similar to those of Experiment 2. However, in the Other condition, participants were asked to compare the test images with Kate Middleton, answering the question: Is this body thinner or fatter than Kate Middleton? We made sure that all participants were familiar with the appearance of Kate Middleton prior to the experiment. Additionally, at the beginning of the experiment, participants were presented with five full body images of Kate Middleton.

Unlike the first two experiments which involved only a thin adapting stimulus, Experiment 3 included both a thin (BMI 13) and a fat adaptor (BMI 35). The experiment therefore consisted of four parts: Baseline and Adaptation, each repeated twice, once with a thin and once with a fat adaptor. Each of these four parts was further divided in four blocks of 36 trials (two blocks per Self/Other condition, in ABAB order, counterbalanced across participants). To further reduce the length of the experiment, initial adaptation was shortened to $1 \mathrm{~min}$, the top-up adaptation to $4 \mathrm{~s}$, and the initial blank screen to $250 \mathrm{~ms}$. Participants took a 10-min break between the two adaptation procedures (i.e., thin and fat) to allow the effect of adaptation to wear off. The order of thin/fat adaptation was counterbalanced across participants.

\section{Results and Discussion}

The results from Experiment 3 are shown in Figure 3. After adaptation to a thin body, in the Self condition, the mean perceived BMI dropped from $23.8(\mathrm{SD}=2.6)$ at pre-test to $21.0(\mathrm{SD}=3.0)$ after adaptation. In the Other (i.e., Kate Middleton) condition, perceived BMI dropped from 21.97 $(\mathrm{SD}=1.7)$ at pre-test to $19.11(\mathrm{SD}=1.24)$ after adaptation. As in the first two experiments, clear aftereffects were apparent in both the Self condition (mean change: 2.78, SD: 1.71), $t(17)=6.89, p<0.001, d_{\mathrm{z}}=1.63$, and the Other (i.e., Kate Middleton) condition (mean change: 2.86 , SD: 1.39$), t(17)=8.76$, $p<0.001, d_{z}=2.07$. Similar aftereffects were also found after adaptation to a fat body. In the Self condition, the mean perceived BMI increased from $22.6(\mathrm{SD}=2.3)$ at pre-test to $25.4(\mathrm{SD}=2.4)$ after adaptation. In the Other condition,

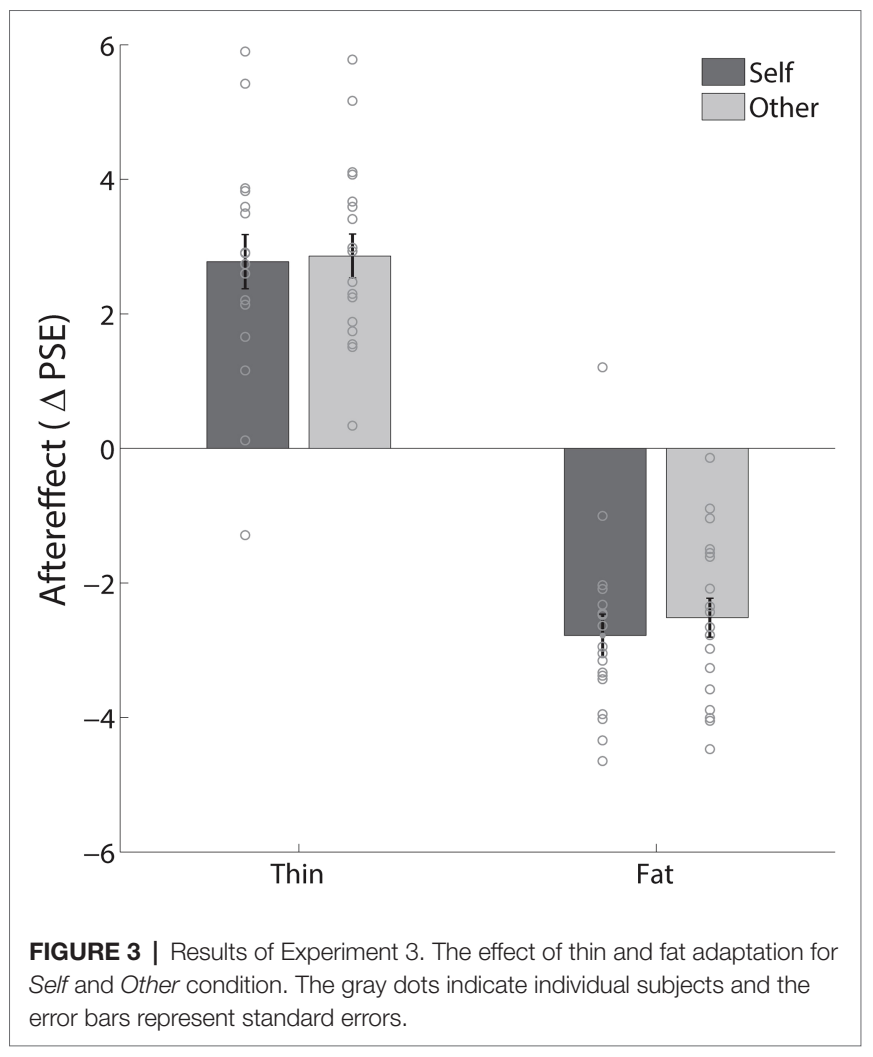

perceived BMI increased from $21.5(\mathrm{SD}=1.6)$ at pre-test to $24.0(\mathrm{SD}=1.8)$ after adaptation. There were clear increases in judged BMI in the Self condition (mean change: -2.78 , SD: 1.34), $t(17)=-8.76, p<0.001, d_{z}=2.07$, and the Other condition (mean change: -2.51 , SD: 1.23 ), $t(17)=-8.67$, $p<0.001, d_{z}=2.04$. Thus, clear aftereffects were found for both thin and fat adapting stimuli, both for judgments of one's own body and of Kate Middleton's body.

A $2 \times 2 \times 2$ repeated measures ANOVA with factors condition (Self/Other), adaptation (Baseline/Adaptation), and adapting body type (thin/fat) was performed on the PSEs. We found no main effect of adaptation, $F(1,17)=0.22, p=0.644, \eta_{\mathrm{p}}^{2}=0.01$. There was, however, a main effect of adapting body type, $F(1,17)=102.34, p<0.001, \eta_{\mathrm{p}}^{2}=0.86$, and an interaction between adaptation and adapting body type, $F(1,17)=187.26$, $p<0.001, \eta_{\mathrm{p}}^{2}=0.92$, showing that adaptation to thin vs. fat bodies produced strong effects in opposite directions. We tested this assumption using two-tailed paired $t$ tests directly comparing results of adaptation sessions to thin vs. fat: $t(17)=-11.78$, $p<0.0001, d_{z}=-2.72$ for the Self condition, and $t(17)=-12.25$, $p<0.0001, d_{\mathrm{z}}=-2.89$ for the Other condition. We also found a main effect of Self/Other condition, $F(1,17)=11.65, p=0.003$, $\eta_{\mathrm{p}}^{2}=0.41$, clearly indicating that participants in the study perceived their bodies as different from Kate Middleton's (overall as fatter). The interaction between the Self/Other condition and the adapting body type (thin/fat) was also significant, $F(1,17)=5.76, p=0.028, \eta_{\mathrm{p}}^{2}=0.25$. However, again there was no interaction between adaptation and Self/Other condition, $F(1,17)=0.306, p=0.587, \eta_{\mathrm{p}}^{2}=0.018$, and no interaction 
between all three factors, $F(1,17)=0.132, p=0.721, \eta_{\mathrm{p}}^{2}=0.08$. This indicates that adaptation affects participants' judgments of themselves in the same way it affected judgments of Kate Middleton's body. As in Experiments 1 and 2, there was a positive correlation between the magnitude of the aftereffects in the two conditions, though it did not differ significantly from $0: r=0.33, p=0.18$ for the thin adaptation and $r=0.22$, $p=0.38$ for the fat adaptation.

In the thin adaptation, there was a correlation between participants' own BMI and the baseline PSEs in the Self condition: $r=0.66, p=0.003$, but not in the Other condition: $r=0.20, p=0.426$. Similarly, after adaptation, there was a correlation between participants' own BMI and PSEs in the Self condition: $r=0.77, p<0.001$, but not in the Other condition: $r=0.25, p=0.308$. In fat adaptation, there was a correlation between participants' own BMI and the baseline PSEs in the Self condition: $r=0.72, p<0.001$, but not in the Other condition: $r=0.03, p=0.906$. Similarly, after adaptation there was a correlation between participants' own BMI and PSEs in the Self condition: $r=0.70, p=0.001$, but not in the Other condition: $r=0.05, p=0.845$.

Again, the results of the Bayesian paired $t$-test comparing the magnitude of the aftereffects in two conditions showed that data support the null hypothesis for both, thin adaptor, $\mathrm{BF}_{(0,0.7)}=0.25$, and fat adaptor, $\mathrm{BF}_{(0,0.7)}=0.30$.

\section{GENERAL DISCUSSION}

Body dissatisfaction is a prevalent problem in modern societies (Grogan, 2017). Media are often blamed for creating unrealistic, unhealthy body ideals that can shape our beliefs and attitudes (Derenne and Beresin, 2006). Recently, it has also been suggested that exposure to thin ideals can influence the way we actually perceive our own bodies. Several studies (Hummel et al., 2012b; Brooks et al., 2016) proposed visual adaptation as a model of media influences on one's own body image. Our results replicate previous results showing that visual adaptation to extreme body types affects body image judgments. Critically, however, virtually identical effects were found for judgments of other people's bodies. This suggests that adaption does not have specific effects on the participant's body image. We suggest instead that adaptation may have affected body types of judgment by changing visual perception of the test stimuli. Here, we showed that adaptation affects judgments about one's own body in a similar way as judgments about other people's bodies, both when asking about typical bodies (Experiment 1), or about a specific other person's body (Experiments 2 and 3). Importantly, we found a main effect of Self/Other condition in Experiments 2 and 3 , indicating that people were indeed performing different tasks when comparing the test stimuli with either their own body or bodies of others.

Our findings are consistent with the results of previous studies that reported transfer of body size aftereffects between different identities (Hummel et al., 2012b; Brooks et al., 2016). The authors of these studies suggest that their results reflect perceptual bias similar to those evoked by exposure to thin ideals in Western culture and that this bias may contribute to the development of body image disorders. It is true that for visual adaptation to constitute an experimental model of body image distortion, some overlap of the representation of self and others is required to allow the transfer of aftereffects from media images to the perception of one's own body. However, if visual adaptation to bodies truly modulates one's own body image, then it should also differ from the general effect adaptation has on all images of bodies. If both the item being tested (own body) and the probe (other bodies) are equally affected by adaptation, the relative difference between them should not change. If all bodies are equally affected, adaptation to extreme body types cannot serve as a sufficient explanation for own body image distortion. If, however, adaptation affects perception of one's own body and other bodies differently, it may suggest that it affects higher level representation of one's own body and not only the experience of the visual image. Here, we found equally strong aftereffects for judgments about one's own body and other people's bodies. Thus, our results provide no evidence that body size adaptation has an effect that shows specificity to one's body image.

Brooks et al. (2016) showed stronger body size aftereffects when the identity between the adaptor and test stimuli matched, regardless of whether both corresponded to images of the participant's own body or the body of an unknown other. Nonetheless, they also found some transfer in body size aftereffects between identities, so that adaptation to a fat or thin body of an unknown other affected the perception of images of their own body at test, and vice versa. These effects suggest a partial dissociation of the neural mechanisms encoding body size for self and other. However, as noted by the authors themselves, the use of only two identities ("own body" and "the body of an unfamiliar other") does not allow to rule out the possibility that the observed effects are not specific to the self, but rather would have been observed regardless of the two specific identities used. In the present study, we address this issue by requesting judgments about the same test stimuli throughout, but using as criteria different internal representations (i.e., an image of the body self or the body of another person), varying, therefore, the context. Interestingly, Winkler and Rhodes (2005) found that attractiveness aftereffects were observed following adaptation to extremely thin bodies but not following adaptation to fat bodies, whereas no such asymmetry was found when participates were asked to judge the perceived normality of the test image. This asymmetry suggests that strength of adaptation aftereffects in some cases may be mediated by the context of the task. Furthermore, de la Rosa et al. (2014) found modulation of action adaptation aftereffects across two conditions that had identical adapting and test stimuli but differed with respect to the information that was provided prior to the adaptation, i.e., the social context. However, in our study, the context of the specific judgment being made (i.e., about one's own body or about someone else's) did not affect the magnitude of the adaptation aftereffects.

There are some clear limitations of the present study. Our study reports null findings, which have an ambiguous 
status in the field, not allowing to draw very strong conclusions. However, we found consistent results across experiments and in all three experiments, we report Bayes factors that provided moderate support in favor of the null hypothesis. Furthermore, the use of only a single identity stimuli meant that adaptation in our study was specific to this identity (see Brooks et al., 2016). Future studies should further investigate this topic using multiple identities that share only the property of adiposity.

Our study investigated short-term effects of relatively short adaptation period (a couple of minutes). It is possible that longer term adaptation affects body representations in a way that goes beyond pure visual perception. One of the main characteristics of both low-level and high-level perceptual aftereffects is that their magnitude depends on the length of adaptation (Leopold et al., 2005). Recent research on lower level visual adaptation aftereffects has suggested that there may be qualitatively distinct mechanisms that underlie adaptation over very short-term and longer term time scales (e.g., Vul et al., 2008; Bao and Engel, 2012; Bao et al., 2013). It is therefore possible that long-term exposure to extreme body types could produce a different pattern of results to those we found in this study. Future studies should examine whether prolonged visual adaptation can cause aftereffects in another modality, related to body representation, e.g., touch.

Our results also do not allow us to distinguish whether the aftereffects we report reflect changes in the actual perception of body stimuli or higher level decisional processes used to make judgments about these stimuli. Recent work has suggested that many effects previously interpreted as perceptual may actually reflect decisional processes (e.g., Morgan et al., 2011; Firestone and Scholl, 2013). The psychophysical methods used in this study, like nearly all other studies of "high-level"

\section{REFERENCES}

Anstis, S., Verstraten, F. A. J., and Mather, G. (1998). The motion aftereffect. Trends Cogn. Sci. 2, 111-117. doi: 10.1016/S1364-6613(98)01142-5

Bao, M., and Engel, S. A. (2012). Distinct mechanisms for long-term contrast adaptation. Proc. Natl. Acad. Sci. USA 109, 5898-5903. doi: 10.1073/ pnas.1113503109

Bao, M., Fast, E., Mesik, J., and Engel, S. A. (2013). Distinct mechanisms control contrast adaptation over different timescales. J. Vis. 13:14. doi: 10.1167/13.10.14

Barlow, H. B., and Hill, R. M. (1963). Evidence for a physiological explanation of the waterfall illusion. Nature 200, 1345-1347. doi: 10.1038/2001345a0

Ben-Tovim, D. I., and Crisp, A. H. (1984). The reliability of estimates of body width and their relationship to current measured body size among anorexic and normal subjects. Psychol. Med. 14, 843-846. doi: 10.1017/S0033291700019814

Ben-Tovim, D. I., Walker, M. K., Murray, H., and Chin, G. (1990). Body size estimates: body image or body attitude measure? Int. J. Eat. Disord. 9, 57-67. doi: 10.1002/1098-108X(199001)9:1<57::AID-EAT2260090107>3.0.CO;2-S

Boothroyd, L. G., Tovee, M. J., and Pollet, T. V. (2012). Visual diet versus associative learning as mechanisms of change in body size preferences. PLoS One 7:e48691. doi: 10.1371/journal.pone.0048691

Bould, H., Carnegie, R., Allward, H., Bacon, E., Lambe, E., Sapseid, M., et al. (2018). Effects of exposure to bodies of different sizes on perception of and satisfaction with own body size: two randomised studies. R. Soc. Open Sci. 5:171387. doi: $10.1098 /$ rsos.171387

Brainard, D. H. (1997). The psychophysics toolbox. Spat. Vis. 10, 433-436. doi: $10.1163 / 156856897 X 00357$ adaptation aftereffects, do not allow these interpretations to be distinguished (cf. Storrs, 2015).

\section{DATA AVAILABILITY STATEMENT}

The datasets generated for this study are available on request to the corresponding author.

\section{ETHICS STATEMENT}

This study was carried out in accordance with the recommendations of the ethics board of the Department of Psychological Sciences, Birkbeck, University of London, with written informed consent from all subjects. All subjects gave written informed consent in accordance with the Declaration of Helsinki. The protocol was approved by the ethics board of the Department of Psychological Sciences, Birkbeck, University of London.

\section{AUTHOR CONTRIBUTIONS}

$\mathrm{KA}, \mathrm{EA}$, and ML designed the study and wrote the manuscript. KA collected the data. KA analyzed the data under supervision of EA and ML.

\section{FUNDING}

This research was supported by European Research Council grant (ERC-2013-StG-336050) under FP7 to ML.

Brooks, K., Clifford, C. W. G., Stevenson, R. J., Mond, J., and Stephen, I. D. (2018). The high level basis of body adaptation. R. Soc. Open Sci. 5, 1-9. doi: $10.1098 /$ rsos. 172103

Brooks, K. R., Mond, J. M., Stevenson, R. J., and Stephen, I. D. (2016). Body image distortion and exposure to extreme body types: contingent adaptation and cross adaptation for self and other. Front. Neurosci. 10:334. doi: 10.3389/ fnins.2016.00334

Challinor, K. L., Mond, J., Stephen, I. D., Mitchison, D., Stevenson, R. J., Hay, P., et al. (2017). Body size \& shape misperception and visual adaptation: an overview of an emerging research paradigm. J. Int. Med. Res. 45, 2001-2008. doi: $10.1177 / 0300060517726440$

Cornelissen, P. L., Cornelissen, K. K., Groves, V., McCarty, K., and Tovée, M. J. (2018). View-dependent accuracy in body mass judgements of female bodies. Body Image 24, 116-123. doi: 10.1016/j.bodyim.2017.12.007

Cornelissen, P. L., Toveé, M. J., and Bateson, M. (2009). Patterns of subcutaneous fat deposition and the relationship between body mass index and waistto-hip ratio: implications for models of physical attractiveness. J. Theor. Biol. 256, 343-350. doi: 10.1016/j.jtbi.2008.09.041

de la Rosa, S., Streuber, S., Giese, M., Bülthoff, H. H., and Curio, C. (2014). Putting actions in context: visual action adaptation aftereffects are modulated by social contexts. PLoS One 9:86502. doi: 10.1371/journal.pone.0086502

Derenne, J. L., and Beresin, E. V. (2006). Body image, media, and eating disorders. Acad. Psychiatry 30, 257-261. doi: 10.1176/appi.ap.30.3.257

Firestone, C., and Scholl, B. J. (2013). “Top-down" effects where non should be found: the El Greco fallacy in perception research. Psychol. Sci. 25, 38-46. doi: 10.1177/0956797613485092 
Fox, C. J., and Barton, J. J. S. (2007). What is adapted in face adaptation? The neural representations of expression in the human visual system. Brain Res. 1127, 80-89. doi: 10.1016/j.brainres.2006.09.104

Garner, D. M., Garfinkel, P. E., Stancer, H. C., and Moldofsky, H. (1976). Body image disturbances in anorexia nervosa and obesity. Psychosom. Med. 38, 327-336. doi: 10.1097/00006842-197609000-00005

Gibson, J. J., and Radner, M. (1937). Adaptation, after-effect and contrast in the perception of tilted lines. I. Quantitative studies. J. Exp. Psychol. 20, 453-467. doi: 10.1037/h0059826

Glauert, R., Rhodes, G., Byrne, S., Fink, B., and Grammer, K. (2009). Body dissatisfaction and the effects of perceptual exposure on body norms and ideals. Int. J. Eat. Disord. 42, 443-452. doi: 10.1002/eat.20640

Groesz, L. M., Levine, M. P., and Murnen, S. K. (2002). The effect of experimental presentation of thin media images on body satisfaction: a meta-analytic review. Int. J. Eat. Disord. 31, 1-16. doi: 10.1002/eat.10005

Grogan, S. (2017). Body image: Understanding body dissatisfaction in men, women and children. 3rd Edn. New York, NY: Routledge.

Guardia, D., Conversy, L., Jardri, R., Lafargue, G., Thomas, P., Dodin, V., et al. (2012). Imagining one's own and someone else's body actions: dissociation in anorexia nervosa. PLoS One 7, 1-9. doi: 10.1371/journal.pone.0043241

Health Survey for England (2003). National Centre for Social Research and University College London. Department of Epidemiology and Public Health. Colchester, Essex: UK Data Archive.

Health Survey for England (2016). NHS Digital. National Centre for Social Research, University College London, Department of Epidemiology and Public Health. Available from: https://digital.nhs.uk/data-and-information/publications/statistical/ health-survey-for-england/health-survey-for-england-2016

Horndasch, S., Heinrich, H., Kratz, O., Mai, S., Graap, H., and Moll, G. H. (2015). Perception and evaluation of women's bodies in adolescents and adults with anorexia nervosa. Eur. Arch. Psychiatry Clin. Neurosci. 265, 677-687. doi: 10.1007/s00406-015-0603-3

Hummel, D., Grabhorn, R., and Mohr, H. M. (2012a). Body-shape adaptation cannot be explained by adaptation to narrow and wide rectangles. Perception 41, 1315-1322. doi: 10.1068/p7197

Hummel, D., Rudolf, A. K., Brandi, M.-L., Untch, K.-H., Grabhorn, R., Hampel, H., et al. (2013). Neural adaptation to thin and fat bodies in the fusiform body area and middle occipital gyrus: an fMRI adaptation study. Hum. Brain Mapp. 34, 3233-3246. doi: 10.1002/hbm.22135

Hummel, D., Rudolf, A. K., Untch, K. H., Grabhorn, R., and Mohr, H. M. (2012b). Visual adaptation to thin and fat bodies transfers across identity. PLoS One 7, 1-6. doi: 10.1371/journal.pone.0043195

JASP Team (2017). JASP (Version 0.8.1.1) [Computer software].

Leopold, D. A., Rhodes, G., Muller, K.-M., and Jeffery, L. (2005). The dynamics of visual adaptation to faces. Proc. R. Soc. B 272, 897-904. doi: 10.1098/ rspb. 2004.3022

Longo, M. R. (2015). Implicit and explicit body representations. Eur. Psychol. 20, 6-15. doi: 10.1027/1016-9040/a000198

Longo, M. R., Azañón, E., and Haggard, P. (2010). More than skin deep: body representation beyond primary somatosensory cortex. Neuropsychologia 48, 655-668. doi: 10.1016/j.neuropsychologia.2009.08.022

McCollough, C. (1965). Color adaptation of edge-detectors in the human visual system. Science 149, 1115-1116. doi: 10.1126/science.149.3688.1115

Mizes, J. S. (1992). The body image detection device versus subjective measures of weight dissatisfaction: a validity comparison. Addict. Behav. 17, 125-136. doi: 10.1016/0306-4603(92)90017-P

Morgan, M., Fillenberger, B., Raphael, S., and Solomon, J. A. (2011). Observers can voluntarily shift their psychometric functions without losing sensitivity. Atten. Percept. Psychophysiol. 74, 185-193. doi: 10.3758/s13414-011-0222-7

Øverås, M., Kapstad, H., Brunborg, C., Landrø, N. I., and Lask, B. (2014). Memory versus perception of body size in patients with anorexia nervosa and healthy controls. Eur. Eat. Disord. Rev. 22, 109-115. doi: 10.1002/ erv.2276

Pond, S., Kloth, N., McKone, E., Jeffery, L., Irons, J., and Rhodes, G. (2013). Aftereffects support opponent coding of face gender. J. Vis. 13:16. doi: $10.1167 / 13.14 .16$

Rhodes, G., Jeffery, L., Watson, T. L., Clifford, C. W. G., and Nakayama, K. (2003). Fitting the mind to the world: face adaptation and attractiveness aftereffects. Psychol. Sci. 14, 558-566. doi: 10.1046/j.0956-7976.2003.psci_1465.x

Rouder, J. N., Speckman, P. L., Sun, D., Morey, R. D., and Iverson, G. (2009). Bayesian $\mathrm{t}$ tests for accepting and rejecting the null hypothesis. Psychon. Bull. Rev. 16, 225-237. doi: 10.3758/PBR.16.2.225

Schilder, P. (1935/1950). The image and appearance of the human body. New York, NY: International Universities.

Schneider, N., Frieler, K., Pfeiffer, E., Lehmkuhl, U., and Salbach-Andrae, H. (2009). Comparison of body size estimation in adolescents with different types of eating disorders. Eur. Eat. Disord. Rev. 17, 468-475. doi: 10.1002/ erv.956

Slade, P. D., and Russell, G. F. (1973). Awareness of body dimensions in anorexia nervosa: cross-sectional and longitudinal studies. Psychol. Med. 3, 188-199. doi: $10.1017 /$ S0033291700048510

Stephen, I. D., Sturman, D., Stevenson, R. J., Mond, J., and Brooks, K. R. (2018). Visual attention mediates the relationship between body satisfaction and susceptibility to the body size adaptation effect. PLoS One 13:e0189855. doi: 10.1371/journal.pone.0189855

Storrs, K. R. (2015). Are high-level aftereffects perceptual? Front. Psychol. 6:157. doi: $10.3389 /$ fpsyg.2015.00157

Thompson, J. K., Berland, N. W., Linton, P. H., and Weinsier, R. L. (1986). Utilization of a self-adjusting light beam in the objective assessment of body distortion in seven eating disorder groups. Int. J. Eat. Disorder. 5, 113-120. doi: 10.1002/1098-108X(198601)5:1<113::AID-EAT2260050110>3.0.CO;2-D

Tiggemann, M., and McGill, B. (2004). The role of social comparison in the effect of magazine advertisements on women's mood and body dissatisfaction. J. Soc. Clin. Psychol. 23, 23-44. doi: 10.1521/jscp.23.1.23.26991

Tovée, M. J., Emery, J. L., and Cohen-Tovée, E. M. (2000). The estimation of body mass index and physical attractiveness is dependent on the observer's own body mass index. Proc. R. Soc. Lond. B 267, 1987-1997. doi: 10.1098/ rspb. 2000.1240

Vul, E., Krizay, E., and MacLeod, D. I. (2008). The McCollough effect reflects permanent and transient adaptation in early visual cortex. J. Vis. 8:4. doi: $10.1167 / 8.12 .4$

Watson, A. B., and Pelli, D. G. (1983). QUEST: a Bayesian adaptive psychophysical method. Percept. Psychophys. 33, 113-120. doi: 10.3758/BF03202828

Webster, M. A., Kaping, D., Mizokami, Y., and Duhamel, P. (2004). Adaptation to natural facial categories. Nature 428, 557-561. doi: 10.1038/nature02420

Winkler, C., and Rhodes, G. (2005). Perceptual adaptation affects attractiveness of female bodies. Br. J. Psychol. 96, 141-154. doi: 10.1348/000712605X36343

Wohlgemuth, A. (1911). On the aftereffect of seen movement. Br. J. Psychol. 1(Monograph Suppl. 1), 1-117.

Conflict of Interest: The authors declare that the research was conducted in the absence of any commercial or financial relationships that could be construed as a potential conflict of interest.

Copyright (c) 2019 Ambroziak, Azañon and Longo. This is an open-access article distributed under the terms of the Creative Commons Attribution License (CC $B Y)$. The use, distribution or reproduction in other forums is permitted, provided the original author(s) and the copyright owner(s) are credited and that the original publication in this journal is cited, in accordance with accepted academic practice. No use, distribution or reproduction is permitted which does not comply with these terms. 